\title{
Feasibility Study of Voice Shutter
}

\author{
Masaharu Nishimura* \\ *Department of Mechanical and Aerospace Engineering, Tottori University, Tottori, Japan \\ E-mail: mnishimura@mech.tottori-u.ac.jp, Tel: +81-857-31-5765
}

\begin{abstract}
Telephone voice is annoying in public spaces like trains and cafés. The speaking voice was tried to be attenuated by using an active noise control technique. It is called 'Voice Shutter'. Two types of voice shutter, which are open type and closed type, are proposed in this paper. A closed type voice shutter controlled by a feed forward method was manufactured and tested. As the results, the voice shutter was proved to be feasible, although it was too large for practical use, in this stage.
\end{abstract}

\section{INTRODUCTION}

Active noise control (ANC) has been developed and successfully applied to actual machines, such as airconditioning ducts, engine mufflers, ear protectors, car cabins, noise barriers and so forth. Recently some unique applications have been developed such as for reducing snore noise [1], quieting infant incubators [2] and protecting human ears from MRI (Magnetic Resonance Imaging) noise [3]. The author et al have been developing AAS-Window (Active Acoustic Shielding) which is an open wind without transmitting sound [4]. These applications can be established only by active methods, not by passive methods. These are considered to be important application fields of the ANC techniques.

On the other hand, there is a problem that telephone voice is annoying in such public spaces as trains and cafés. This is an issue of manner. However it is convenient if we can use a mobile phone in the public space without leaking the voice in the neighborhood. If we cover our mouth perfectly by some caps, we cannot speak any more. Therefore some vent holes are necessary for speaking easily. The voice should be shielded not to leak through the holes. Any passive techniques are difficult to solve this problem because of their size and weight.

In this paper, Active Noise Control (ANC) techniques were tried to be applied to the above shielding. We call this system 'Voice Shutter'. The concepts of Voice Shutter were proposed and the feasibility was studied by a trial manufacturing and some experiments. As the first step of this research, our concern was concentrated on how much voice shielding performance could be obtained and whether this system disturbed the telephone communication ability or not. The size and weight of Voice Shutter were not concerned in this stage.

\section{CONCEPT OF VOICE SHUTter}

Fig. 1 shows the conceptual configurations of Voice Shutter. Type A is a closed type which is pressed against the mouth with no gap and has some vent holes with active sound shielding The leak voice can be controlled by either a feed forward method or a feedback method. Type B is an open type which is used apart from the mouth. Breath and voice are leaked through the gap between the voice shutter and the mouth. The leaked voice is controlled by a feedback method. The open type is our final target. However it is supposed to be difficult to be developed. Therefore, as the first step, the feasibility of the closed type with a feed forward control is examined in this paper.

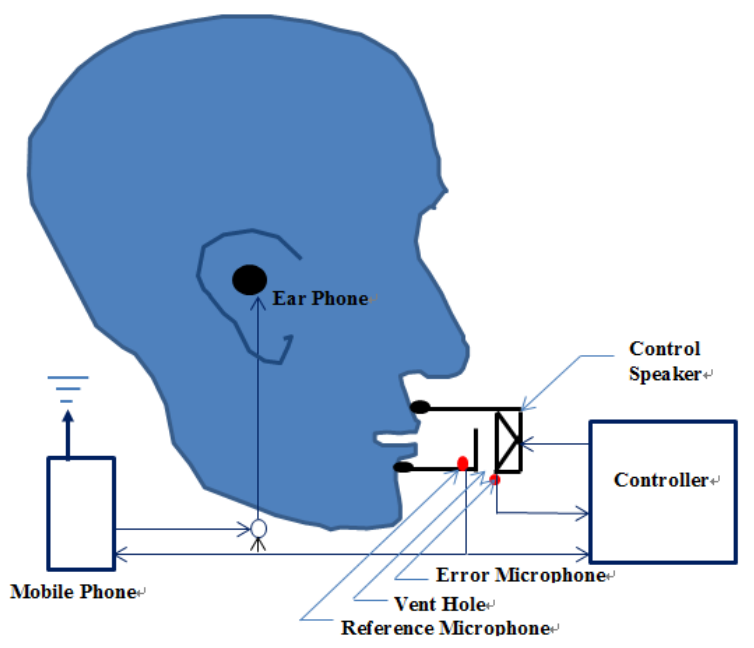

Type A : Closed type

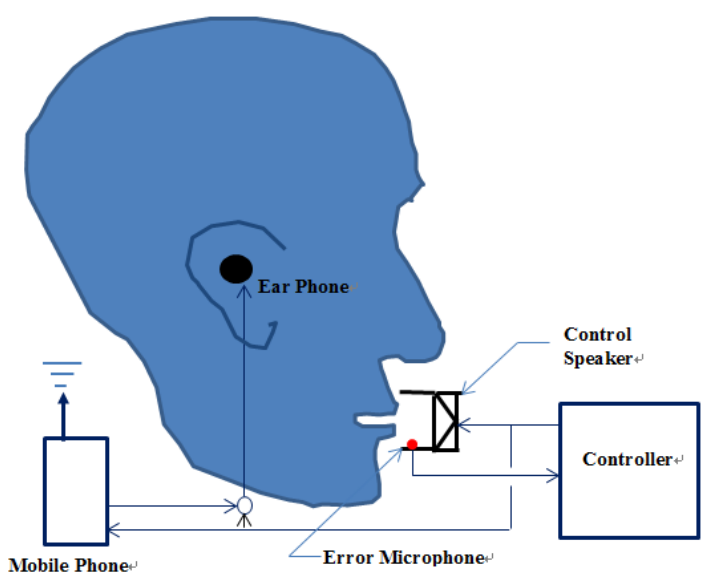

Type B : Open type

Fig. 1 Conceptual configuration of Voice Shutter 


\section{PRELIMINARY EXPERIMENTS}

\section{A. Effect of Perfect Voice Shutter}

The human voice is considered to be mainly radiated from a mouth. However some parts of the voice are also supposed to be radiated from nose holes and the surface of face and neck. Then, the sound reducing effect was ensured when the voice from a mouth was perfectly attenuated.

Fig. 2 shows the photos of the experiment. In this case, the voice from a mouth was perfectly reduced by a long pipe with acoustic lining. The measured sound spectra of the voice [a] at $1 \mathrm{~m}$ apart from the mouth with/without the sound reducing pipe are shown in Fig. 3. It is clear that if the voice from a mouth is perfectly attenuated, the sound around the person is largely reduced by over $20 \mathrm{~dB}$ above $500 \mathrm{~Hz}$. Therefore, the voice shutter attenuating the voice from a mouth is expected to be useful if it is developed.
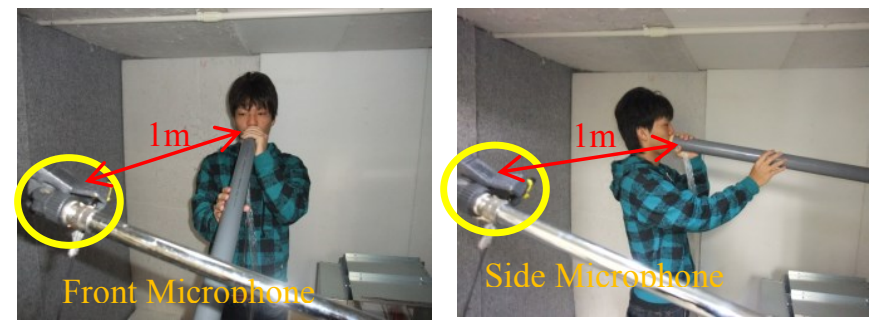

Fig. 2 Photos of the preliminary experiments ensuring the sound reducing effect of the perfect voice shutter

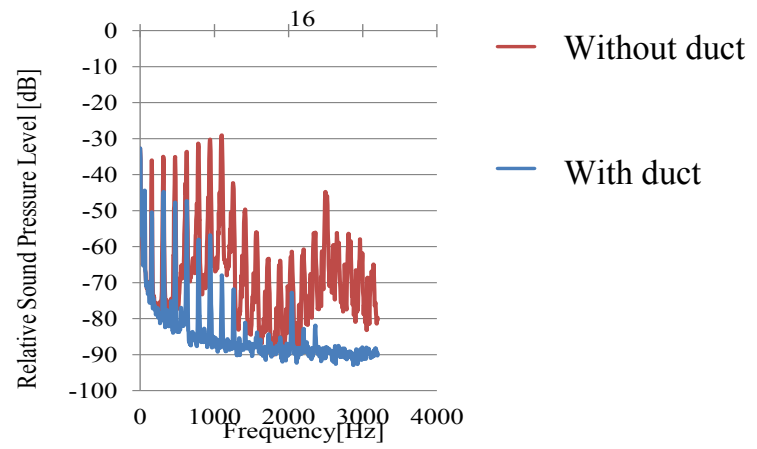

(a) Front microphone

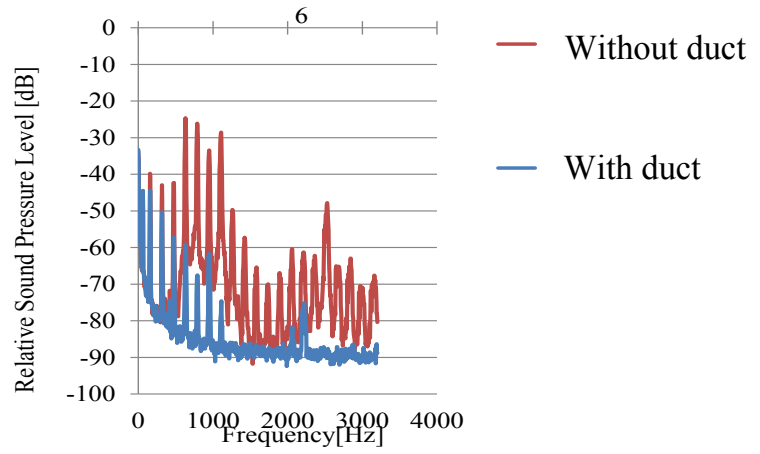

(b) Side microphone

Fig. 3 Sound reducing effects of the perfect voice shutter

\section{B. Size of the Vent Hole}

As mentioned above, if we cover our mouth perfectly by a cap, we cannot speak any more. Therefore the voice shutter must have some vent holes for speaking easily. It was tested how large size of a hole was necessary using some caps with various sizes of holes. As the results, it was found that a hole of at least $10 \mathrm{~mm}$ diameter was enough for speaking easily.

\section{Feasibility of Feed Forward Control}

When we apply the feed forward control method to a voice shutter, it is necessary to shorten the distance between the reference microphone and the control speaker, in order to make a voice shutter compact. However the distance is determined based on the causality between the primary sound and the control sound. We adopted the over sampling method to shorten the distance. The delay of the control system is mainly caused by the sampling delay, the delay of antialiasing filters and the delay of speaker response itself. The higher the sampling frequency is, antialiasing filters with the higher cut off frequency can be used and the shorter the delay of signal processing is. We used a flat speaker as a control speaker because of its quick response.

Fig. 4 shows the preliminary test arrangement for examining how short the distance can be. This is a single channel ANC system with a controller worked by the ordinary Filtered-X-LSM algorithm. Redec EX-Tool was used as a controller. Extremely high sampling frequency such as $48 \mathrm{kHz}$ was used compared with the ordinary ANS system. The control speaker was PROTRO-NCFR. Fig. 5 shows noise reducing effects at the error microphone. It is found that in the case of the sampling frequency $f_{s}=48 \mathrm{kHz}$, the distance between the reference microphone and the control speaker $\mathrm{L}=20 \mathrm{~mm}$ is feasible to reduce the error signal, although it is not feasible in the case of $f_{s}=20 \mathrm{kHz}$. This means that the higher sampling is useful for improving the causality. $\mathrm{L}=20 \mathrm{~mm}$ is amazingly short distance which has not been realized in the previous works. In the next section, we designed a voice shutter whose $\mathrm{L}=50 \mathrm{~mm}$, considering some margin.

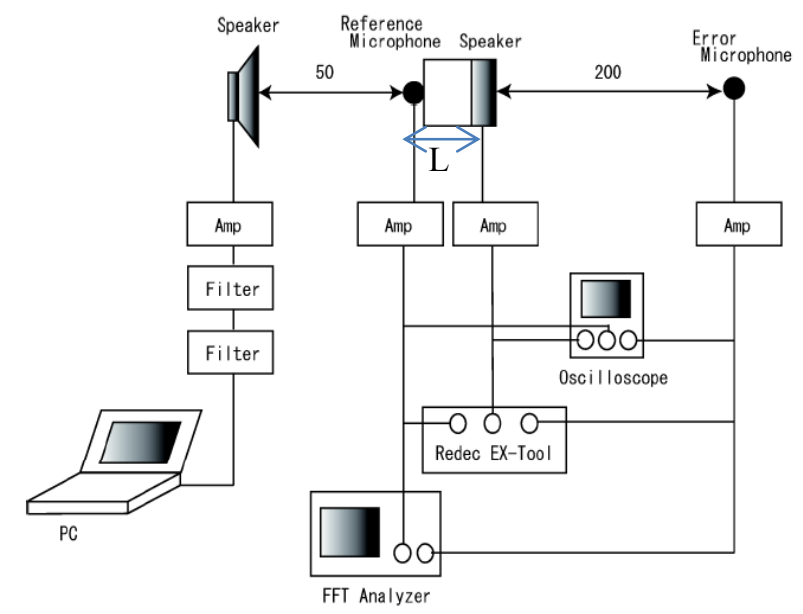

Fig. 4 Preliminary test arrangement for examining how short the distance $\mathrm{L}$ is possible 


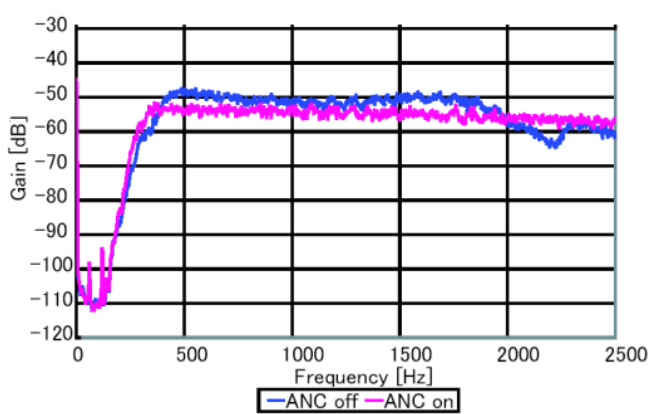

(a) $\mathrm{L}=20 \mathrm{~mm}, f_{s}=20 \mathrm{kHz}$

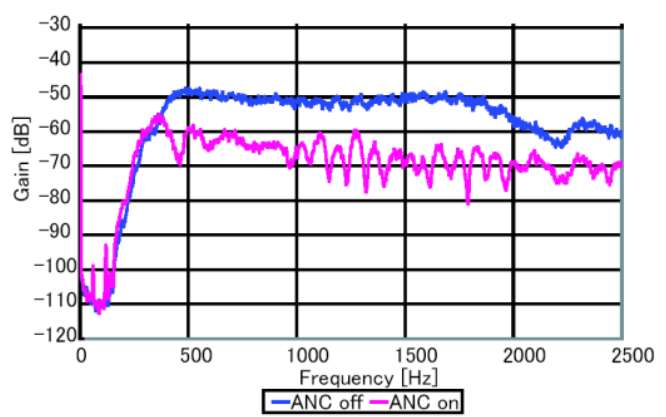

(b) $\mathrm{L}=20 \mathrm{~mm}, f_{s}=48 \mathrm{kHz}$

Fig. 5 Sound spectra at the error microphone in the preliminary test shown in Fig.4

\section{Trial Manufacturing of Voice ShUtter}

A closed type voice shutter was designed and manufactured for the trial experiments. Fig. 6 shows the schematic drawings and photos of the voice shutter. A PVC (Polyvinyl chloride) pipe of $71 \mathrm{~mm}$ inner diameter and $168 \mathrm{~mm}$ length was used as a casing. It is divided two rooms (forward and backward) by a separate plate with a vent hole of $10 \mathrm{~mm}$ dia..

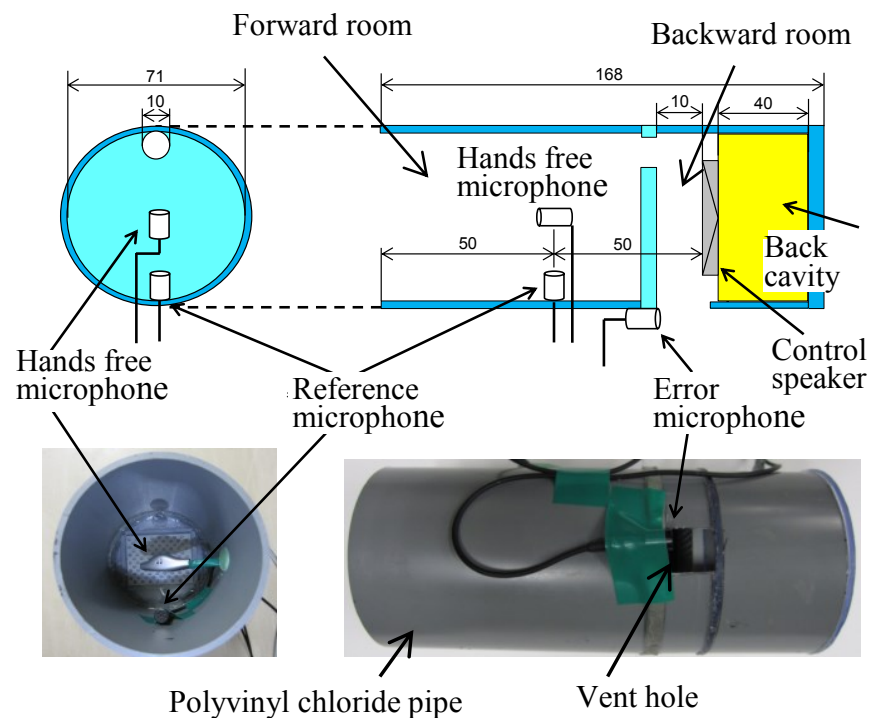

Fig. 6 Schematic drawings and photos of the voice shutter.
In the forward room a reference microphone and a hands free microphone are installed. Here the latter was used only to connect easily to a mobile phone. Generally the former can be used both for the reference signal and for the input signal to the mobile phone as shown in Fig. 1. In the backward room a flat speaker (PROTRO-NCFR, $47 \mathrm{~mm} \times 57 \mathrm{~mm}$ ) is installed as a control speaker with a back cavity filled with sound absorbing material. An error microphone is set just outside of the exit vent hole. The distance $\mathrm{L}$ is about $50 \mathrm{~mm}$ in this case.

\section{EXPERIMENTS WITH SPEAKER VOICE}

\section{A. Experimental Set Up and Conditions}

At first, we performed some experiments that reduced the speaker voice radiated from a small cone-type speaker instead of real voice. Fig. 7 shows the experimental set up.

The controller was Redec EX-Tool and was worked by the ordinary Filtered-X-LMS algorithm whose control parameters were as follows.

- Sampling frequency: $f_{s}=48 \mathrm{kHz}$

- Cut off frequency of the anti-aliasing filters: $f_{c}=20 \mathrm{kHz}$

- Filter tap length: Adaptive filter: 400, Error path filter: 256 The howling cancelling filter was not used. The adaptive filter was converged by using band limited white noise from $100 \mathrm{~Hz}$ to $5 \mathrm{kHz}$ as the primary noise and it was fixed after converged. The experiments attenuating the speaker voice were performed using the fixed filter. The test sentence was 'kono kawa wo wataru to mamonaku niigataken desu' (in Japanese).

The effect of voice shutter was evaluated by two points of view. First is how much it can attenuate the leaking voice which is measured at $500 \mathrm{~mm}$ apart from the mouth (Monitor point A). Second is how much it distorts the telephone voice received by a person talking with. It was measured at the vicinity of the receiver (Monitor point B) as shown in Fig. 8.

Experiments were performed in three cases, without V.S. with V.S. (ANC off) and with V.S. (ANC on), and these results were compared with each other. Here V.S. means Voice Shutter.

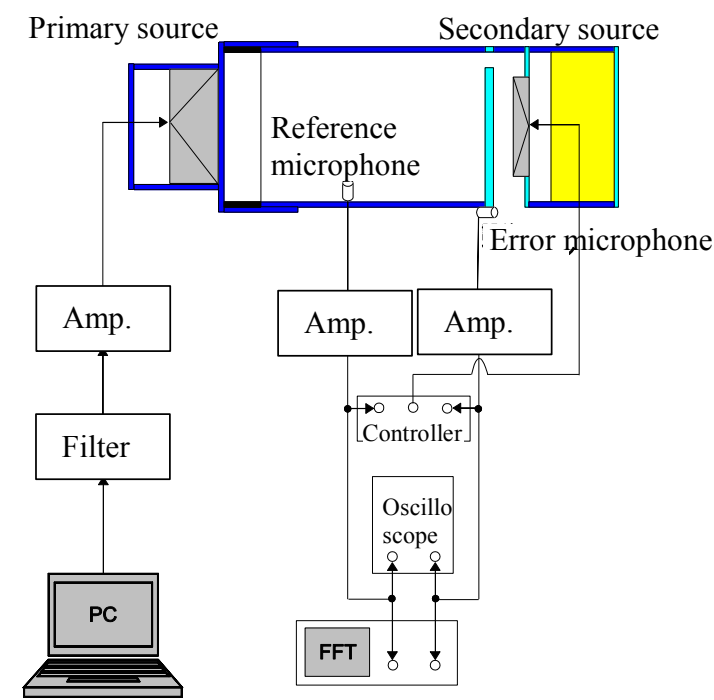

Fig. 7 Experimental set up for speaker voice 

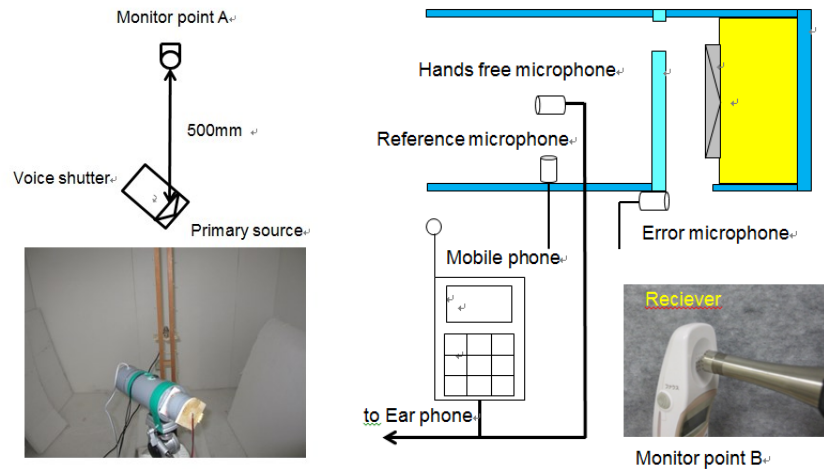

Fig. 8 Sound evaluation points (monitor points A and B)

\section{B. Results and Discussions}

Fig. 9 and 10 show the sound spectra measured at the error microphone and at monitor point A, respectively, in the case of using the band limited white noise as the primary sound. When the voice shutter (ANC off) is attached to the mouth, the radiated sound spectra are attenuated by the passive effect. However there remain some peaks at the resonant frequency of the voice shutter. And ANC can reduce those peaks. It is found that the voice shutter with ANC can attenuate the radiated white noise by roughly $20 \mathrm{~dB}$.

Fig.11 shows sound spectrograms measured at monitor point $\mathrm{A}$ in the case of using the vocal sentence as the primary sound. It is found that some formant components are remained in the case of ANC off. However, the voice shutter (ANC on) can be seen largely to attenuate the vocal sentence. These effects were also ensured by listening.

Fig. 12 and 13 show the results at monitor point B. The received telephone voice was distorted a little but the sentence was recognized clearly.

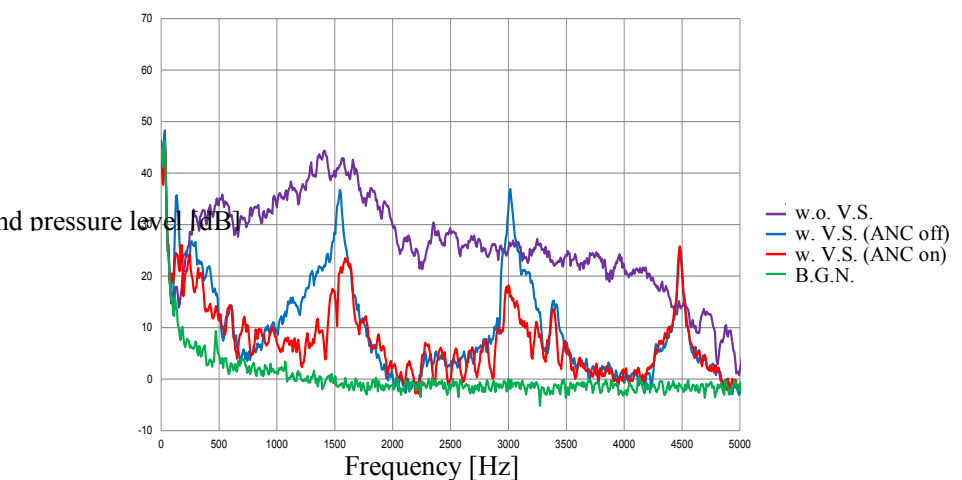

Fig. 10 Sound spectra at monitor point A (White noise)

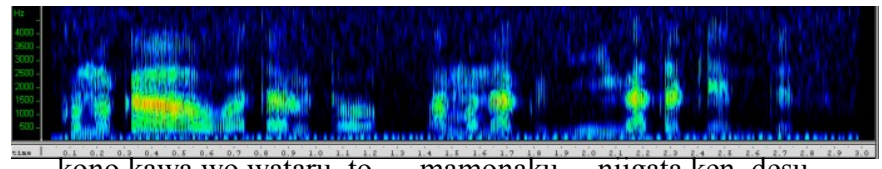

kono kawa wo wataru to mamonaku nigata ken desu

(a) Without Voice Shutter

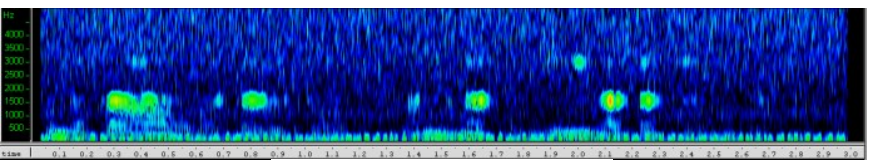

(b) With Voice Shutter (ANC off)

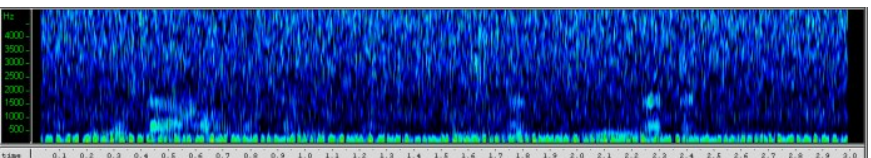

(c) With Voice Shutter (ANC on)

Fig. 11 Sound spectrograms at monitor point A (Speaker voice)

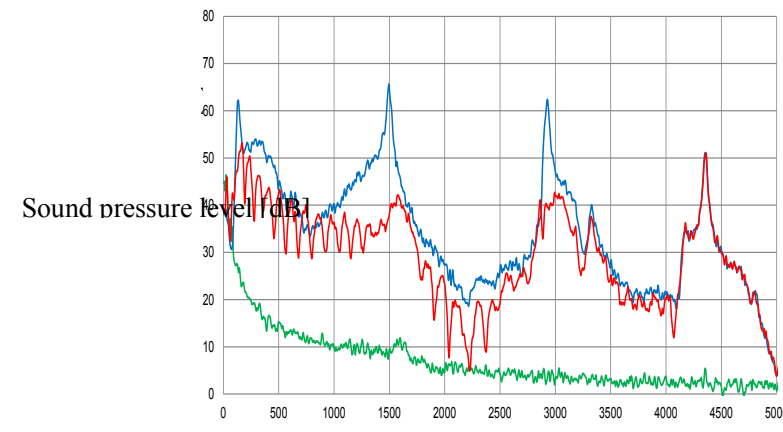

Frequency $[\mathrm{Hz}]$

Fig. 9 Sound spectra at the error microphone (White noise)

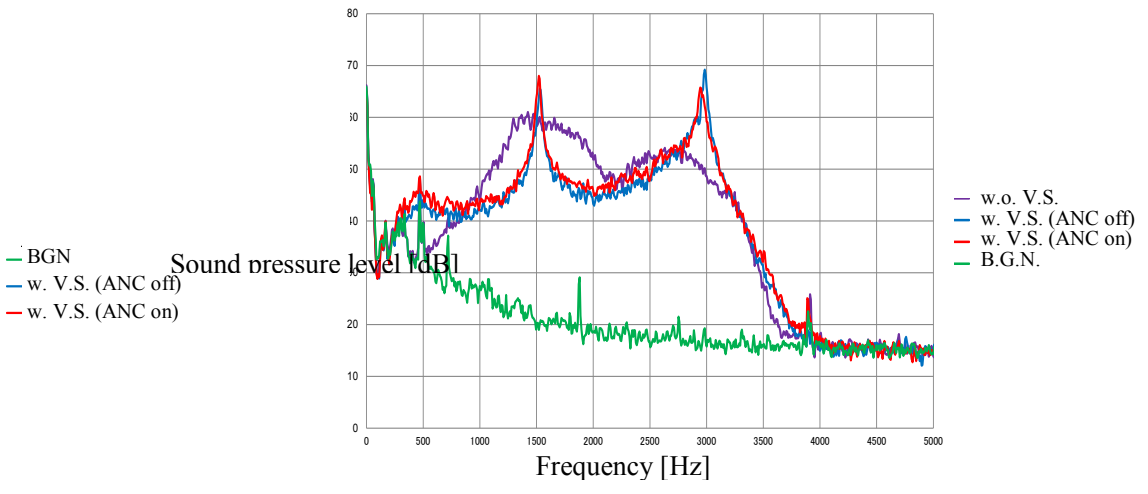

Fig. 12 Sound spectra at monitor point B (White noise) 


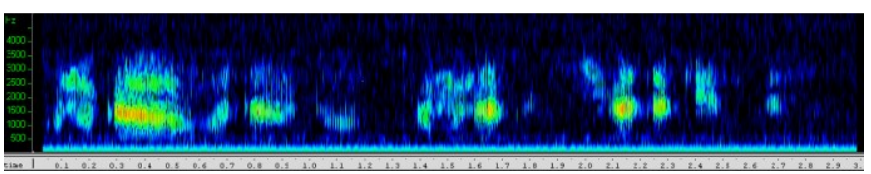

kono kawa wo wataru to mamonaku niigata ken desu (a) Without Voice Shutter

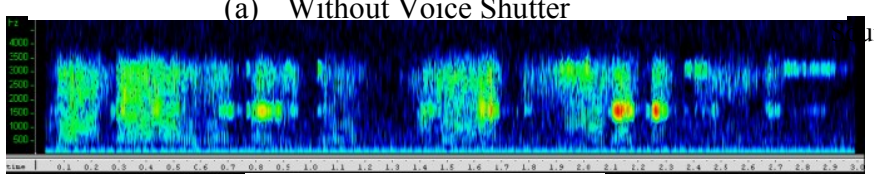

(b) With Voice Shutter (ANC off)

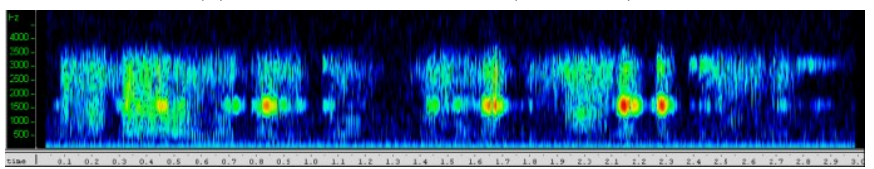

(c) With Voice Shutter (ANC on)

Fig. 13 Sound spectrograms at monitor point B

(Speaker voice)

In order to reduce the resonant peaks of the voice shutter, some sound absorbing materials were inserted in the voice shutter as shown in Fig.14. Obtained results are shown in Figs. 15 - 19. The control conditions are same as the above. These figures show that the resonant peaks are reduced remarkably and the leaked voice was attenuated and almost inaudible at monitor point A even in the case of with the voice shutter (ANC off). Moreover it was perfectly inaudible in the case of ANC on. The sound spectra at monitor point B also became smooth and the received voice became milder.

From the above results the manufactured voice shutter is found to be sufficiently effective for the speaker voice. It seems to have a little over specification and to have a potential to be lighter and smaller.
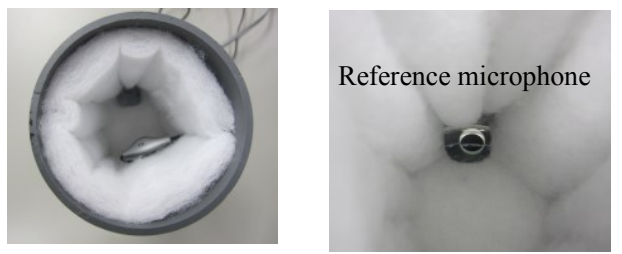

Fig. 14 Inside view of the voice shutter with sound absorbing material

ound pressure

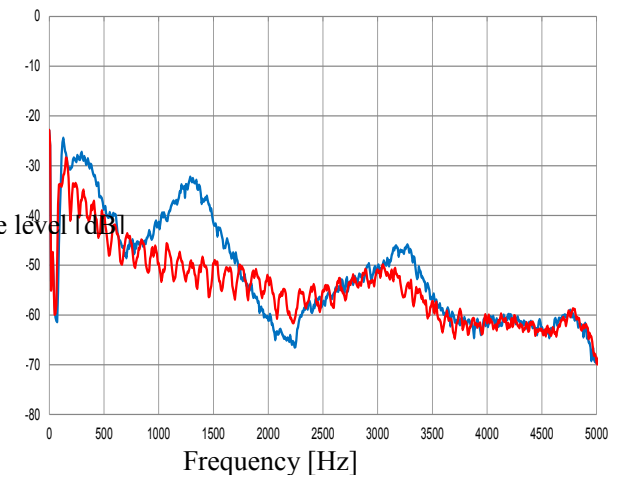

- w. V.S. (ANC off)

- w. V.S. (ANC on)

Fig. 14 Sound spectra at the error microphone (White noise),

V.S. with sound absorbing material

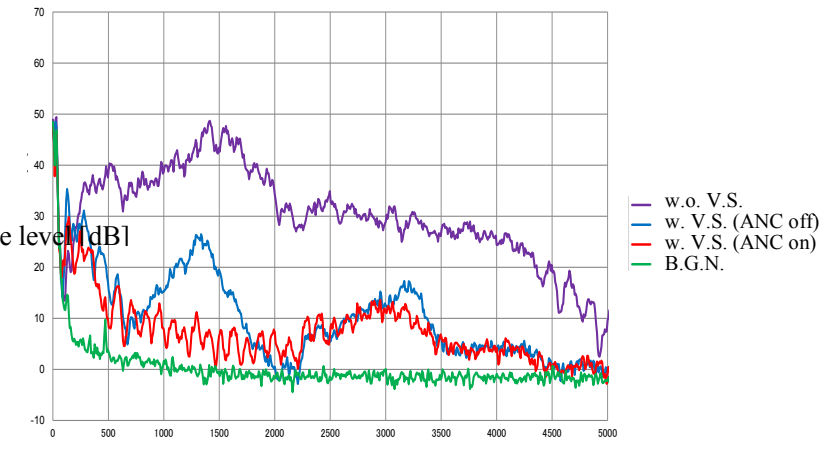

Frequency $[\mathrm{Hz}]$

Fig. 16 Sound spectra at monitor point A (White noise), V.S. with sound absorbing material

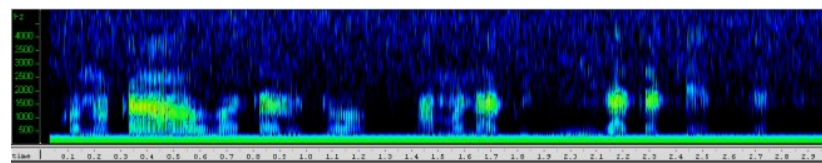

kono kawa wo wataru to mamonaku niigata ken desu

(a) Without Voice Shutter

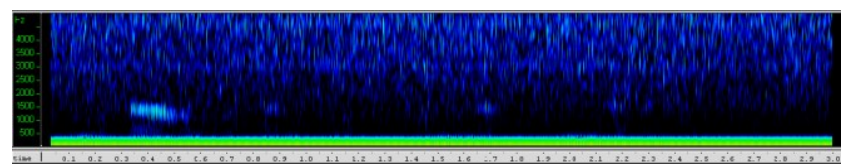

(b) With Voice Shutter (ANC off)

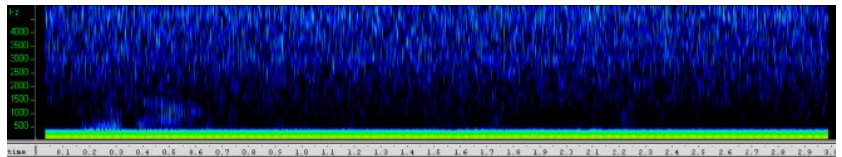

(c) With Voice Shutter (ANC on)

Fig. 17 Sound spectrograms at monitor point A (Speaker voice), V.S. with sound absorbing material

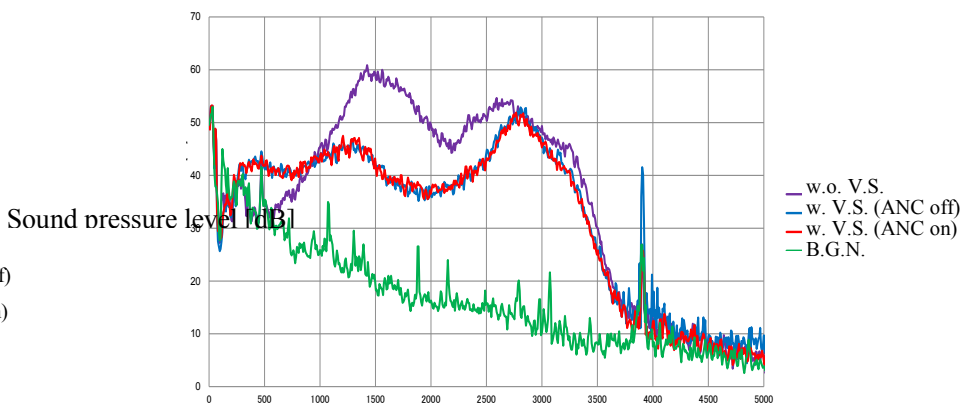

Frequency $[\mathrm{Hz}]$

Fig. 18 Sound spectra at monitor point B (White noise), V.S. with sound absorbing material 


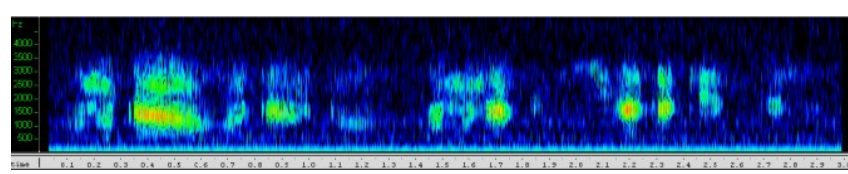

kono kawa wo wataru to mamonaku niigata ken desu (a) Without Voice Shutter

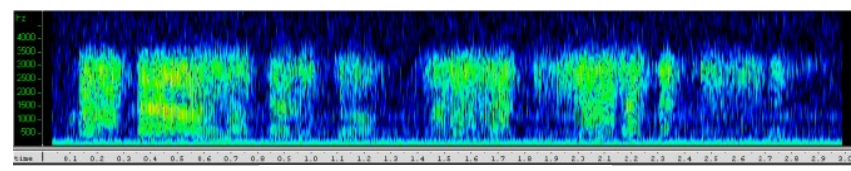

(b) With Voice Shutter (ANC off)

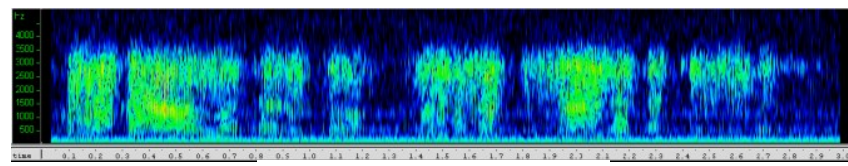

(c) With Voice Shutter (ANC on)

Fig. 19 Sound spectrograms at monitor point B (Speaker voice), V.S. with sound absorbing material

\section{EXPERIMENTS WITH REAL VOICE}

\section{A. Experimental Set Up and Conditions}

Experiments were performed using real voice radiated from a human mouth. A mouth piece was attached to the voice shutter with sound absorbing material as shown in Fig. 20. Control was performed on the same conditions as in the case of speaker voice. The same fixed control filter was also used for the real voice.

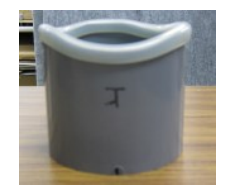

Mouth piece

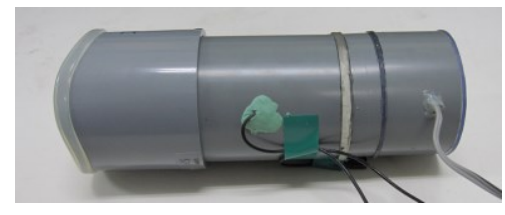

Fig. 20 Voice shutter with mouth piece

\section{B. Results and Discussions}

It was very difficult to adjust the loudness of voice. If we spoke normally, ANC system could not attenuate the voice because of the saturation of microphone signal and insufficient sound radiation ability of the control speaker. However, if we spoke with small voice, the similar effects as in the case of speaker voice was obtained. Moreover the voice shutter was too large and too heavy to carry with us.

These results suggest the following issues.

- Some automatic volume control system is necessary.

- The control speaker should have more effective sound radiation ability.

- In order to make 'Voice Shutter' compact, the distance between the reference microphone and the control speaker ' $L$ ' should be smaller or some feedback control system should be developed.

- In order to make 'Voice Shutter' lighter, some lighter material with high sound insulation effect should be used for the casing.
Concerning on the distance ' $\mathrm{L}$ ', we have already developed a higher speed signal processing technique using FPGA (Field Programmable Gate Array). Using higher sampling frequency $f_{s}=100 \mathrm{kHz}, \mathrm{L}=10 \mathrm{~mm}$ is possible as shown in Fig. 21 [5].

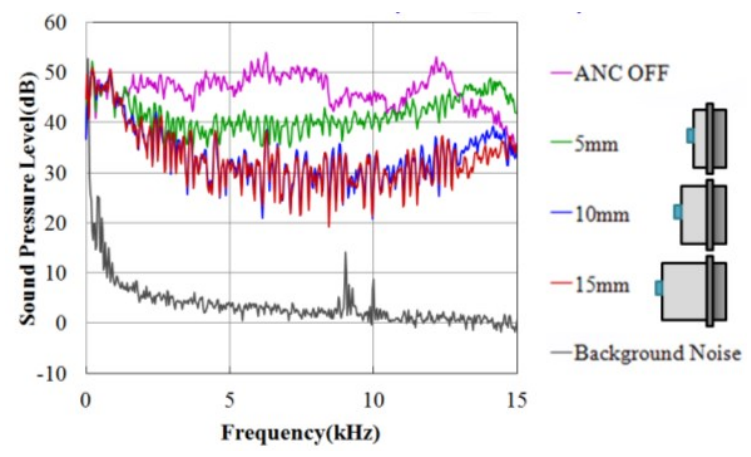

Fig. 21 Noise reducing effect v.s. the distance ' $L$ ', using FPGA controller [5]

\section{CONCLUSIONS}

'Voice Shutter' was proposed in this paper which could reduce the telephone speaking voice by using ANC techniques. The feasibility was examined and the following conclusions were obtained.

(1) A proto-type of voice shutter using the feed forward control was designed, manufactured and tested.

(2) It was proved to be effective for reducing the speaking voice with stable loudness like the speaker voice.

(3) Several issues were made clear for reducing the real voice.

\section{ACKNOWLEDGMENT}

The author acknowledges the students in our laboratory, Mr. Yuichiro Anji, Mr. Keita Ushio, Mr. Koji Shiratori and Mr. Toshihiro Tanaka for their kind assistance.

\section{REFERENCES}

[1] Sen M. Kuo, "Digital signal processing algorithms and implementations on active noise control systems," Proc. of ACTIVE2006, CD-ROM, 2006

[2] Lichuan Liu, Kapila Beemanpally, and Sen M. Kuo, "Multichannel hybrid active noise control systems for infant incubators," Proc. of Internoise2012, CD-ROM, 202

[3] Michal Meller, Maciej Niedzwiecki, Hirofumi Tetsu and Yoshinobu Kajikawa, "Semi-adaptive feedback active control of MRI noise," Proc. of Internoise2011, CD-ROM, 2011

[4] Tatsuya Murao and Masaharu Nishimura, "Basic study on active acoustic shielding," J. of Environment and Engineering, Vol.7, No.1, pp76-91, 2012

[5] Masaharu Nishimura, Kenichi Nishikage, Tatsuya Murao and Nobutaka Wada, "Development of a ANC unit with a set of colocated reference microphone and control speaker," Proc. of JSME Environmental Engineering Symposium 2010, CD=ROM, 2010 (in Japanese) 